\title{
Parametric Design Techniques Applied to Creative Hollow out Product Design with 3D Voronoi Patterns
}

\author{
Hui-Chin Chang \\ Department of Creative Product Design, HungKuo Delin University of Technology, Taiwan \\ Email: chang.hcjang@gmail.com
}

How to cite this paper: Chang, H.-C. (2021) Parametric Design Techniques Applied to Creative Hollow out Product Design with 3D Voronoi Patterns. Journal of Computer and Communications, 9, 32-47. https://doi.org/10.4236/jcc.2021.98003

Received: June 14, 2021

Accepted: August 15, 2021

Published: August 18, 2021

Copyright $\odot 2021$ by author(s) and Scientific Research Publishing Inc. This work is licensed under the Creative Commons Attribution International License (CC BY 4.0).

http://creativecommons.org/licenses/by/4.0/

\section{(c) (i) Open Access}

\begin{abstract}
The creation of hollow out art includes a variety of materials, techniques and categories, its content mostly emphasizes the ancient philosophy performance of the alternation of virtual and real, and Yin-Yang depends on the essence of Chinese culture deduction. If this feature is applied to product design, in addition to emphasizing functional orientation, this traditional arts integration with the new media, will give users a different visual inspire. This thesis is mainly in view of the importance of hollow out art in Chinese cultural heritage, and the 3D hollow out production craft has gradually lost. Therefore, the Delaunay triangle is constructed based on the Convex Hull interpolation algorithm, and the Voronoi Diagram feature is constructed based on the Divide and Conquer algorithm. And with Rhino modeling software as the main body, combined with the application of the parametric plug-in design program $(\mathrm{GH})$, the 3D models of the parametric creative hollow pen holder and the parametric creative hollow lampshade were respectively completed. The traditional craftsmanship is integrated into the modern manufacturing process with innovative techniques, and the Chinese cultural spirit and beauty of nature are successfully connected.
\end{abstract}

\section{Keywords}

Parametric Design, Hollow Out Product, 3D Voronoi Patterns

\section{Preface}

The so-called Parametric Design is to present all the elements of the design process with data. The geometric model is no longer determined by the graphics drawn by paper and pen, but constructing model by means of data and model 
logic directly. Its design and construction method is not as in the modeling software that we are familiar with, in the $3 \mathrm{D}$ working space with elements such as points, lines and planes, intuitively performing modeling, stacking, stretching and other actions to complete the design techniques of geometric modeling. It is like as on a blank paper-like operating platform, and according to the arrangement and interactive links of data to complete the design process. The application of data gives the definitions and rules behind the natural models a suitable stage. The concept of parametric design allows mathematical definitions and even various algorithms to be added to design ideas. Through the combination of data and algorithms, it can create models based on specific logic or mathematical definitions. In the development of digital architecture, there have been many practical cases of algorithms that use natural models for design creation. People want to do "real" natural design ideas, which seems to be realized through parametric design techniques [1].

The historical development of hollow out art is closely related to life, and then it has developed an application that connects the first and the next. As an artistic expression, it is not alone, but is widely used in a variety of materials. Before the art of paper-cutting, there were hollow carvings on leather and silver foil, and then they were widely used in lace, embroidery, printing and dyeing, and ceramics. These artistic styles, all present the common characteristics of hollow out art, and influence, reflect each other, forming the brilliant culture of hollow out art [2].

Hollow out art is often used in the characteristics of different materials, and its production tools are mainly scissors, carving knives and laser hollow out carvings. In terms of the effect of art, it is no longer just to draw patterns, but to show the characteristics of hollows in the material, but also to restrict in the artistic style, and most of them echoes with the pattern. The pattern is played with the material of realizing, emptied, truncated and preserved [3].

The shape, basic composition and applied materials of hollow out art must be an artistic whole, and its artistic language can fully display the infinite content. Different characteristics have been developed in different regions, different areas have different styles, and the structure has simple and complex decoration characteristics. Taking paper as an example, hollow out art has been popular in China for a long time, is widely used, and has various styles. Chinese folk paper-cuts are pure in shape, simple in structure, bright in color, and are often combined with traditional moral stories; the popular "silhouette painting" in the British folks is also called silhouette pattern. It uses black paper to cut out the shadow of an object, and uses black shadows to form the picture. This kind of silhouette also developed into a "silhouette movie" in the future. Folk paper-cuts in Europe are mostly made by nomads and herders. They are mainly symmetrical "folding and cutting". The pictures are rough and powerful, and most of them are geometrically shaped flower decorations [4].

Hollow out the art also often presents the characteristics of arts and crafts that connect the lines. A line connecting refers to the meticulous changes between 
the lines. The specific and image characteristics of the hollowed-out content must be flexibly changed and the characteristics of cutting must be fully utilized to make the real and virtual. Merge with each other to form a sense of beauty. When creating hollow out art, you must correctly understand and use the cutting technique. In the characteristics of the object, you must work hard on the artistic conception, and then give the beautiful characteristics of the line, so that the hollow shape and appearance merge into a beautiful art [5].

The Voronoi graph is a method proposed by the Dutch climatologist A. H. Thiessen to calculate the average rainfall based on the rainfall of discretely distributed weather stations. That is, all adjacent weather stations are connected to form a triangle, and the vertical bisectors of each side of these triangles are made, so that several vertical bisectors around each weather station form a polygon. Use the rainfall intensity of a unique weather station contained in this polygon to represent the rainfall intensity in this polygonal area, and call this polygon a Voronoi figure.

Voronoi Diagrams are composed of a set of continuous polygons composed of vertical bisectors connecting two adjacent points. The characteristics of Voronoi Diagrams are:

- Each Voronoi Diagram contains only one discrete point data.

- The distance from the point in the Voronoi graph to the corresponding discrete point is the closest.

- The distance between the point on the edge of the Voronoi graph and the discrete points on both sides is equal.

Because Voronoi Diagrams have the above characteristics, they can be used for qualitative analysis, statistical analysis, proximity analysis, etc. Polygon elements created by creating Voronoi Diagrams can divide the available space and assign it to the nearest point element. Voronoi Diagrams are sometimes used in place of interpolation operations to generalize a set of sample measurements to the area closest to them. Using Voronoi Diagrams can generalize the measurement values taken from a group of climate measuring instruments to the surrounding area, and quickly build a service area model for a group of shops.

The most typical example of applying this Voronoi Diagram in the design field is the stadium of the National Aquatics Center (also known as the Water Cube) established by China for the 2008 Beijing Olympics. It was constructed in cooperation with design teams such as PTW Architects, CSCEC, CCDI and Arup. Its surface looks like a cube filled with water, and a membrane structure with different areas and different plating points is installed on the outer wall. This membrane structure adopts the bubble type naturally formed from soap foam, also known as the foam structure of Weaire-Phelan Structure, which is similar to a kind of Voronoi Diagram structure. At the same time, Voronoi Diagrams also exist in the markings or structures of nature's animals and plants, such as leaf cells, giraffe markings, dragonfly wing structures and foam structures. This kind of beautiful natural totem will eventually be valued by people [6]. 
This thesis is mainly in view of the importance of hollow out art in the inheritance of Chinese culture, and the crisis of the gradual loss of three-dimensional hollowing production technology. Therefore, it is planned to use the parametric design method to combine the hollow out art with the Voronoi nature totem to connect the spirit of Chinese culture and the beauty of nature. At the same time, through the collocation of digital processing process (by means of $3 \mathrm{D}$ printer to product prototype), the traditional craftsmanship is integrated into the modern manufacturing process with innovative techniques to show a new style different from the past, which not only retains its inherent spirit, but also can continue and promote this ideal of cultural creativity.

\section{Delaunay Triangle Mesh}

The key to establishing Voronoi Diagrams is to reasonably connect discrete data points into a triangulation. This triangulation is called Delaunay triangulation. Therefore, the Delaunay triangulation, which is dual to the Voronoi Diagram, can be applied to construct the triangulation of a planar figure. The Delaunay triangulation has the empty-circle property which has many advantages as compared with the other triangulation approaches. For instance, the maximized the minimum angle of a Delaunay triangulation [7]. More studies of the Delaunay criterion can be found in Aurenhammer's review paper [8].

The basic principle of Delaunay criterion is that any circumcircle passing through three vertices has no vertex inside its interior in the plane, and we say that it has the empty circle property. The property can also be extended to three dimensions in which any circumsphere passing four vertices has no vertex inside its interior.

\section{Delaunay Triangle Mesh Construction Process}

The construction of Delaunay triangulation is also known as the construction of irregular triangulation, which is to construct a triangulation from discrete data points, and to determine which three data points form a triangle, also known as automatically connected triangulation. That is, for $n$ discrete points on the plane, the plane coordinates are $(x i, y i), i=1,2, \ldots, n$, and three similar points form the best triangle, so that each discrete point becomes the vertex of the triangle, As shown in Figure 1.

The result of automatically connecting the triangulation network is the labels
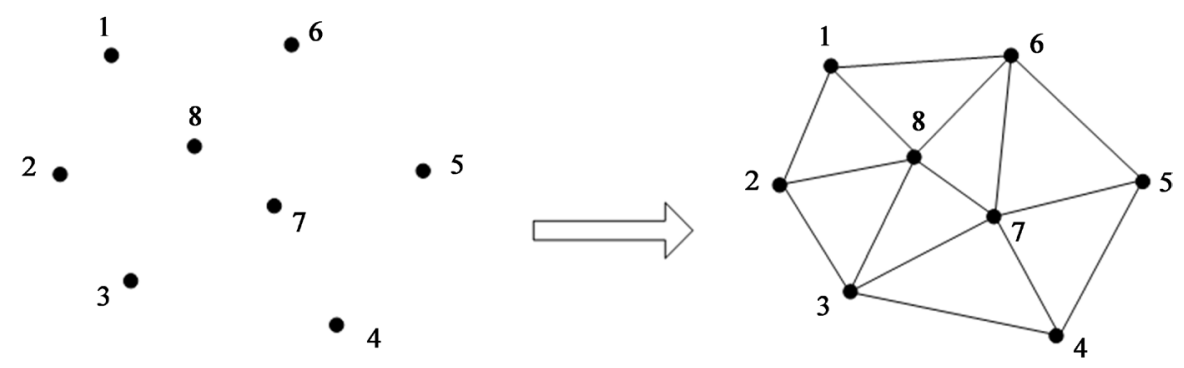

Figure 1. Automatically connect to the triangulation. 
of the three vertices of all triangles, such as: $1,2,8 ; 2,8,3 ; 3,8,7, \ldots$ At the same time, in order to obtain the best triangle, when constructing the triangulation network, the three internal angles of the triangle should be made as acute as possible, which conforms to the criteria for the Delaunay triangle.

1) The circumcircle of any Delaunay triangle cannot contain any other discrete points.

2) Two adjacent Delaunay triangles form a convex quadrilateral. After swapping the diagonals of the convex quadrilateral, the smallest of the six internal angles no longer increases. This property is the minimum angle, maximum criterion.

To achieve this goal, Tsai (1993) proposed a general algorithm for constructing Delaunay triangles in $n$-dimensional Euler space - Convex Hull interpolation algorithm. The calculation steps are as follows:

Step 1: Calculate the four points in the point set satisfying $\min (x-y), \min (x$ $+y), \max (x-y), \max (x+y)$, and form a linked list of points in a counterclockwise direction. These 4 points are the closest points among the discrete points to the 4 corner points of the circumscribed rectangle containing the discrete points. The polygon formed by these 4 points is used as the initial Convex Hull.

Step 2: For each point $I$ on Convex Hull, set its subsequent point to $J$, calculate the distance from all points on the right side of the vector line segmen $t I J$ to $I$, and find the point $K$ with the largest distance.

Step 3: Insert $K$ between $I$ and $J$, and assign $K$ to $J$.

Step 4: Repeat steps 2 and 3 until there is no point on the right side of the line segment $I J$ of the point set.

Step 5: Assign $J$ to $I$, take the subsequent points of $J$, and repeat steps 2,3 , and 4.

Step 6: When there are no discrete points on the right side of the connection between any two adjacent points in Convex Hull, a polygon (Convex Hull) containing all discrete points is formed, as shown in Figure 2.

Step 7: Look for a triangle composed of two adjacent Convex Hull sides in the Convex Hull linked list each time, and the interior and boundary of the triangle do not contain any other points on Convex Hull. After removing this point, a

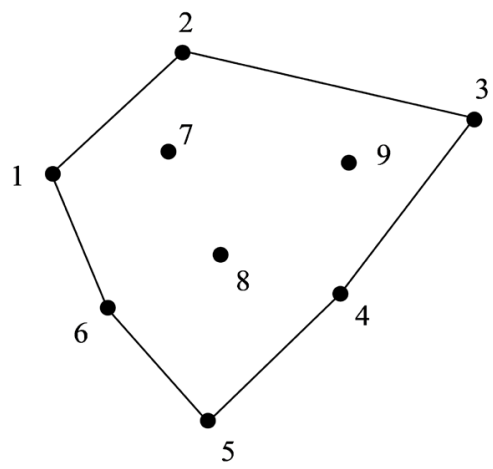

Figure 2. Convex Hull including all discrete points. 
new Convex Hull linked list is obtained. Repeat this process until there are only three discrete points on the Convex Hull list. The last three discrete points in the Convex Hull linked list form a triangle, so the points in Convex Hull form a number of preliminary Delaunay triangles, as shown in Figure 3.

Step 8: After completing the preliminary Delaunay triangle, the remaining discrete points that are not on Convex Hull are divided by point-by-point interpolation until all non-Convex Hull discrete points are inserted, as shown in Figure 4.

And the flowchart of Delaunay triangle constructed as shown in Figure 5.

\section{Voronoi Diagram}

A Voronoi Diagram of a set of "sites" (points) is a collection of region that divides up the plane into disjoint polygons, where the nearest neighbor of any point inside a polygon is the generator of the polygon [9]. Each region corresponds to one of the site, and all the points in one region are closer to the corresponding site than to any other site. A set of points called seeds or generators is specified beforehand. For each seed there will be a corresponding region consisting of all points closer to that generator than to any other [10].

Hence, a Voronoi Diagram for a given data set can be constructed by computing the perpendicular bisectors of the line segments between the pairs of data points. Each data point is then associated with a cell in the Voronoi Diagram.

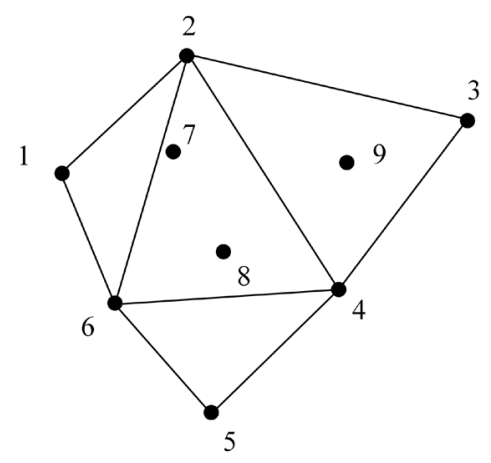

Figure 3. Preliminary Delaunay triangles.

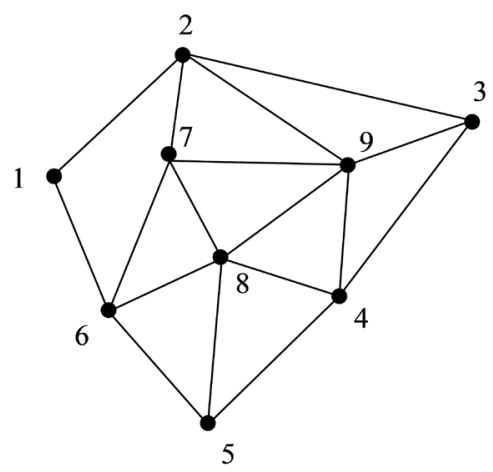

Figure 4. The final Delaunay triangles. 
Calculate the four points in the point set satisfying $\min (x-y), \min (x+y), \max (x-y), \max (x+y)$, and form a linked list of points in a counterclockwise direction as the initial Convex Hull.

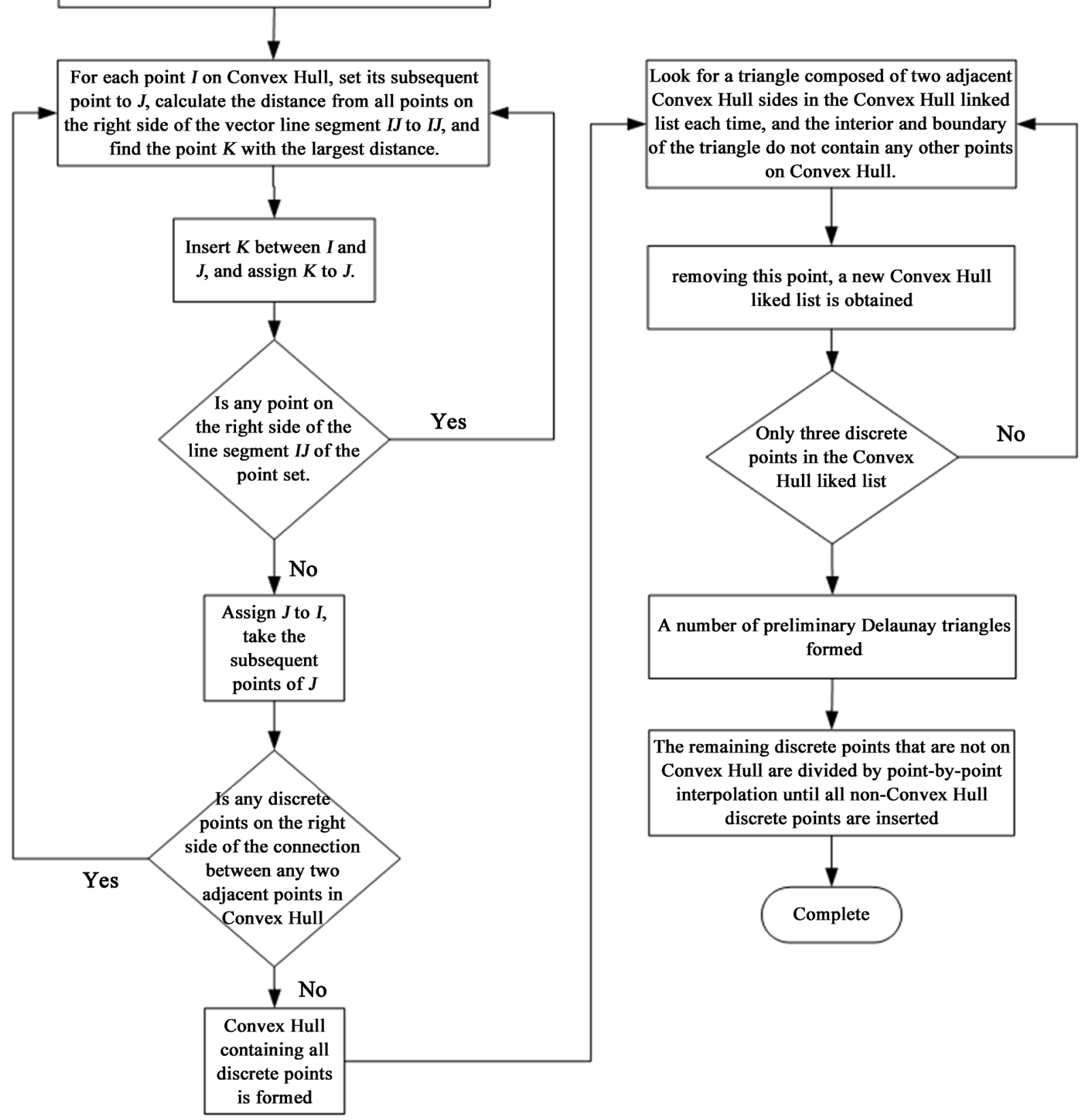

Figure 5. The flowchart of Delaunay triangle constructed.

All of the Voronoi regions are convex polygons. Some of them are infinite they correspond to the sites on the convex hull. The boundary between two adjacent regions is a line segment, and the line that contains it is the perpendicular bisector of the segment joining the two sites. Usually, Voronoi regions meet three at a time at Voronoi points. If three sites determine Voronoi regions that meet at a Voronoi point, the circle through those three sites is centered at that Voronoi point, and there are no other sites in the circle. A Voronoi Diagram of 
$n$ points in a plane under the $L p$-metric, can be constructed on o $(n \log n)$ time.

In short a Voronoi Diagram can be defined as. Let $\mathrm{P}$ be the set of $\mathrm{n}$ distinct site points in the plane. The Voronoi Diagram of $\mathrm{P}$ is the decomposition of the plane into n cells, called Voronoi cell or Dirichlet domain, one for each point. A point lies in the Voronoi cell corresponding to a site point $p_{i}$ if the Euclidean distance $\mathrm{d}\left(q, p_{i}\right)<\mathrm{d}\left(q, p_{j}\right)$, for every $p_{i}$ in $P$, $i$ not equal to $j$. In other words, decomposition of a plane into convex regions all revolving around one generated point and every point in the convex regions closer to the generated point than to any other points is called Voronoi Diagram [11].

\section{Voronoi Diagram Feature Construction Process}

There are literally hundreds of different algorithms for constructing various type of Voronoi Diagrams. In this paper using "Divide and Conquer algorithm" to construct Voronoi Diagram features. The steps are as follows:

Step 1: Scan the point data of the Voronoi graph from left to right and from top to bottom. If the distance between a certain point and the previous scanning point is less than the given proximity tolerance, the point will be ignored in the analysis, as shown in Figure 6.

Step 2: Construct a Delaunay triangulation from the discrete point data, number the discrete points and the constructed triangles, a record which discrete point each triangle is composed of, and record the numbers of all triangles adjacent to each discrete point. As shown in Figure 7.

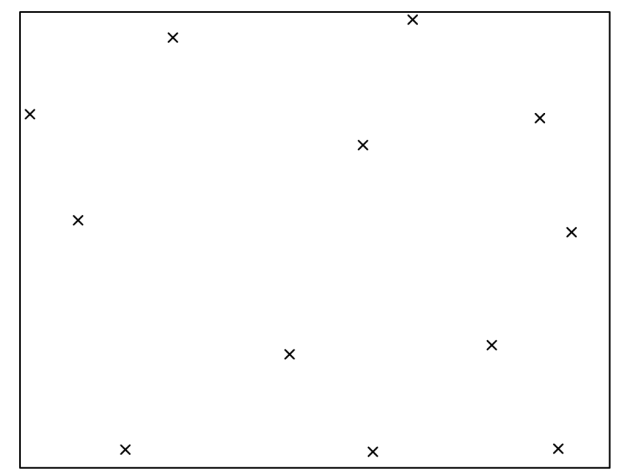

Figure 6. Arrange randomly distributed point data.

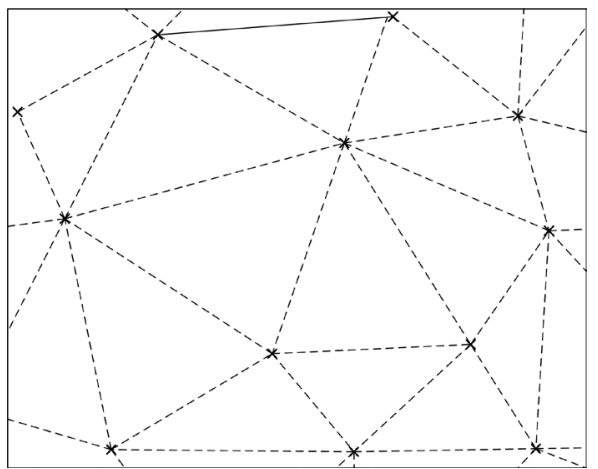

Figure 7. Delaunay Triangulation. 
Step 3: Draw the mid-perpendicular line of each triangle side. These midperpendicular lines form the edges of the Voronoi graph, and the intersection of the mid-perpendicular lines is the vertex of the corresponding Voronoi graph, as shown in Figure 8.

Step 4: The points used for the Voronoi Diagrams will become the anchor points of the corresponding Voronoi Diagrams, as shown in Figure 9.

\section{Modeling Tools}

In recent years, the application of parametric design techniques has gradually become popular. There is more and more CAD (Computer-Aided Design) software based on the concept of parametric and generative design. However, most of the current parametric generative design tools are rarely single the independent software, and most of them are used in the form of plug-in tools on specific CAD platforms. For example, the parametric software "Grasshopper" developed by McNeel can be installed in the same company's Rhinoceros3D modeling software. It can be designed with the $3 \mathrm{D}$ model constructed by Rhino or can be designed in Grasshopper throughout the process. And this article uses Grasshopper as the main design tool. The reason is that Rhinoceros it is easy to operate. It has long been widely used in the industrial design field as one of the modeling tools.

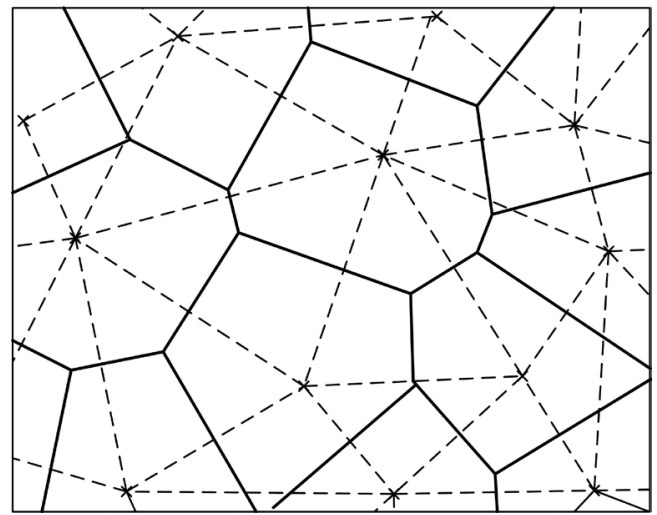

Figure 8. Construct Voronoi Diagrams.

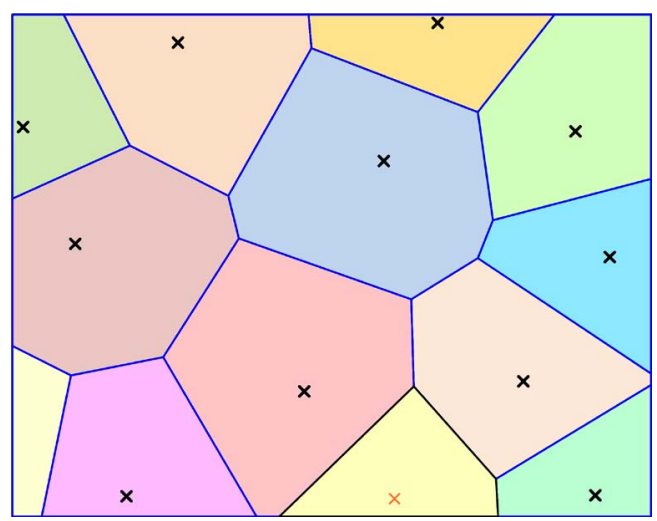

Figure 9. Voronoi Diagrams. 


\subsection{Parametric Design Software - Grasshopper}

The Grasshopper is a graphical parametric design tool developed by the McNeel Corporation since 2007. It is installed in the 3D modeling software Rhinoceros produced by the same company in a plug-in form. Grasshopper is currently a software under work in progress, and many functions are often added and adjusted when the version number is updated. Grasshopper is mainly used to create generative algorithms. 3D geometric models are created through the connection, design of parameters and various calculators. The algorithms in the program are not only limited to the use of numbers, but text, video, audio, and touch applications can all be used as calculations or in the form of parameters. As architectural design is currently the most important field of parametric generative design methods, architects and related learners are currently the main users of Grasshopper, but its gradually improved and increasingly perfect graphical user interface and operation methods allow other fields users also began to pay attention to this software and used it as a tool for parametric design [12].

\subsection{The Modeling Concept of Grasshopper}

Grasshopper (hereinafter referred to as $\mathrm{GH}$ ) is a software that uses the graphics programming language (GPL) as its operating method. Simply put, GH uses Rhinoceros to create basic $2 \mathrm{D}$ and $3 \mathrm{D}$ geometric figures, edits the logic of the subsequent generation of geometric figures through $\mathrm{GH}$, and finally reuses Rhinoceros serves as its platform for presenting calculation results.

For example, in the past, to create a sphere in the Rhinoceros environment, it can be simply divided into several steps. First, draw the section contour line, use the rotation forming command, enter the rotation angle, and finally get the model. The entire construction process is shown in Figure 10(a). But when we need a semicircle, we have to operate the above process again. In the GH environment, we only need to find the most important arithmetic unit in the creation of a spherical surface: the rotating surface. When we understand the parameters required for the rotating surface, the axis of rotation, the section contour, and the angle of rotation, then through the interaction between the arithmetic units, it will automatically construct a complete sphere. The whole construction process is shown in Figure 10(b). Therefore, if we only need a semicircular surface, we can modify the rotation angle parameter immediately.

Taking the gradual change of the sphere as an example again, set the required number parts, and then use the arithmetic progression as the change of the radius parameter to complete the model as shown in Figure 11. When you want to change the requirements, you can quickly generate a new parameter model by adjusting the starting radius of the parameter and increasing the value. In the $\mathrm{GH}$ environment, the relevant operating procedures for constructing $2 \mathrm{~d}$ Voronoi Diagrams are shown in Figure 12. 

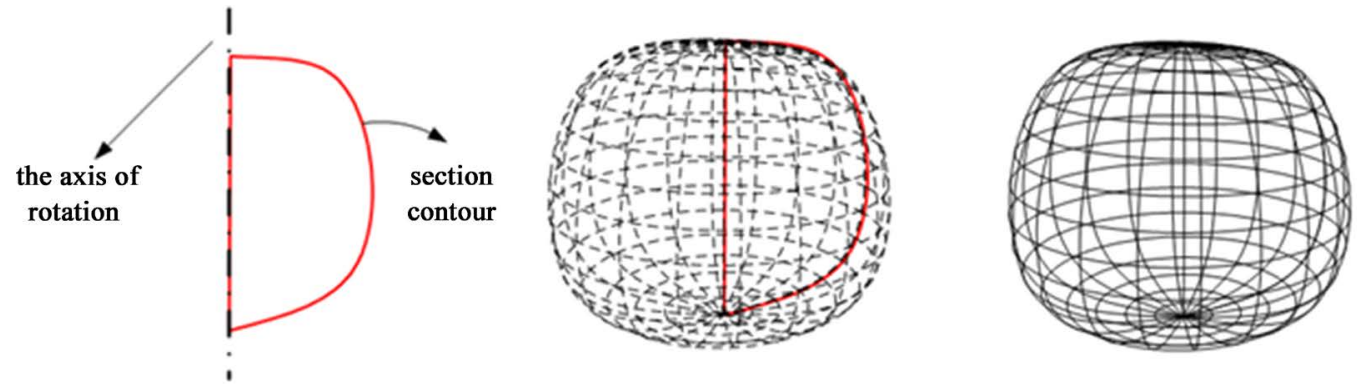

(a) Rhinoceros modeling method

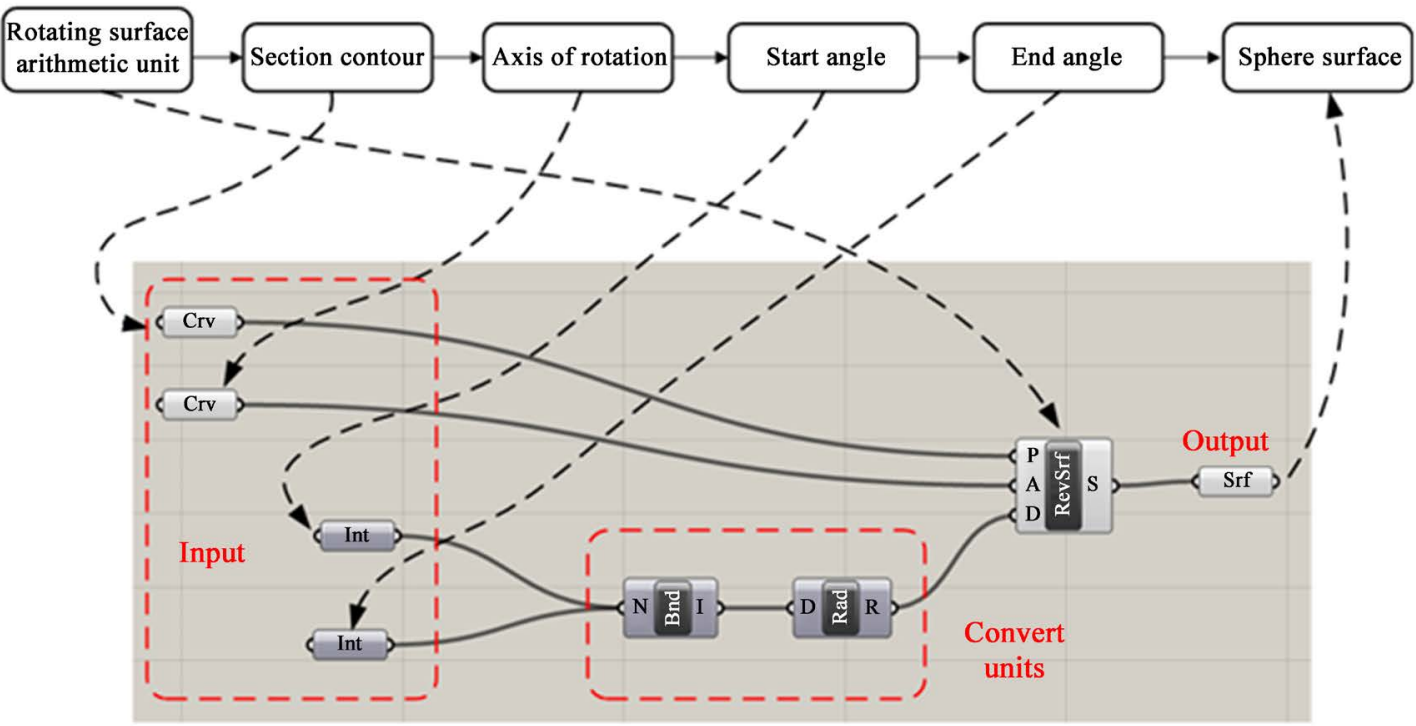

(b) Grasshopper molding method

Figure 10. Differences and correspondence between Rhino and Grasshopper construction models.
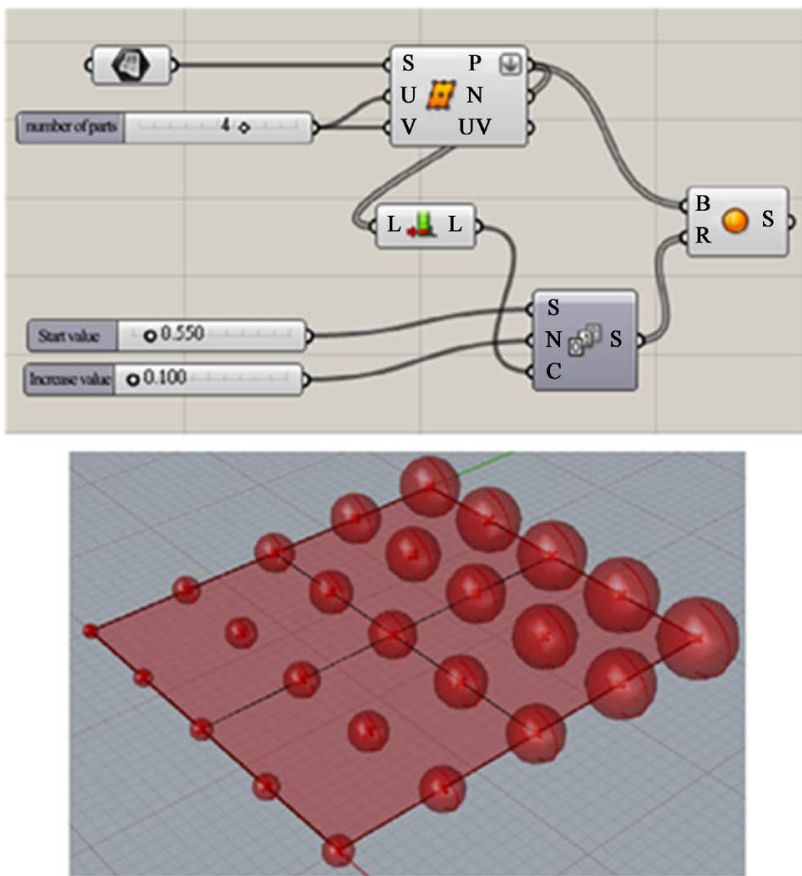

Figure 11. An icon of Grasshopper's construction of a gradient sphere model. 

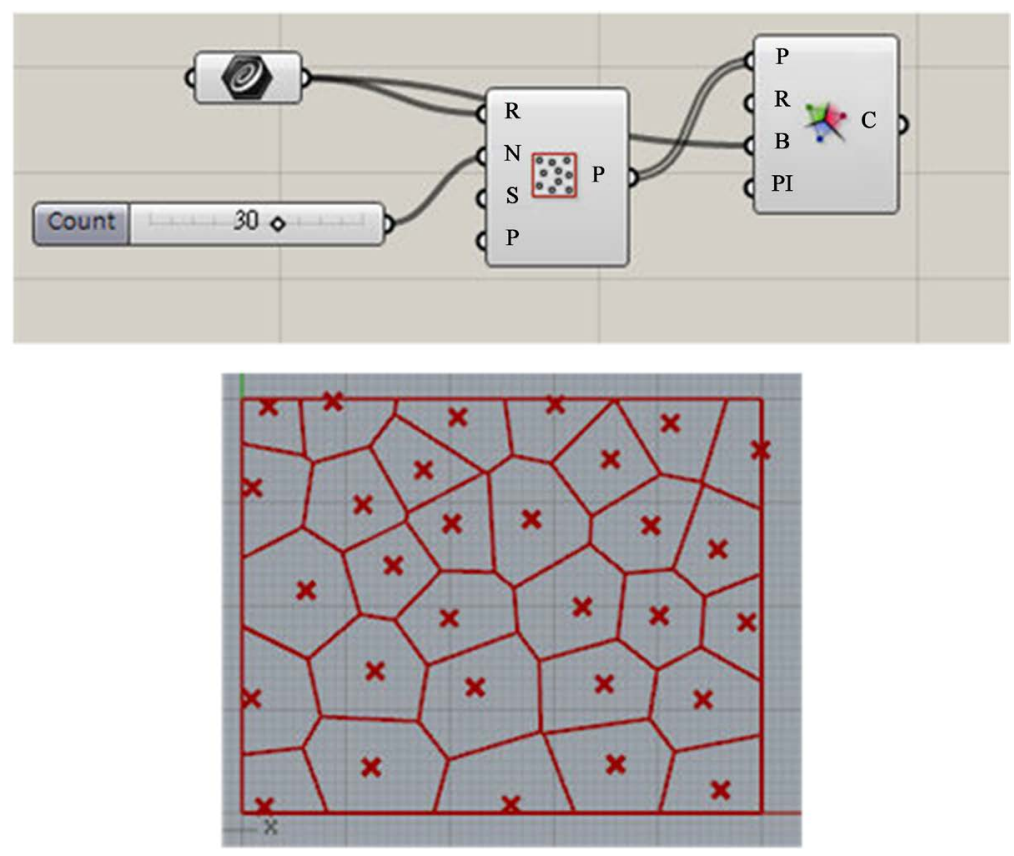

Figure 12. Grasshopper construction of $2 \mathrm{~d}$ Voronoi Diagrams process diagram.

\section{Product Modeling Process}

This thesis takes Rhinoceros modeling software as the main body, combined with the application of the parametric plug-in design program (GH), as the main axis of product creation. Under the premise of the product's own constraints and not focusing on complex design theories, it considers the existing the collocation between the digital processing method and the parametric design, that is, when the design of similar products is completed, the prototype can be made through the $3 \mathrm{D}$ printer. The modeling steps are as follows:

Step 1: In the Rhino environment, construct the main body of the creative product object and the surface object that you want to create 3D Voronoi features.

Step 2: Calculate the smallest volume square that covers this curved object.

Step 3: Generate an appropriate number of randomly distributed points in this volume box.

Step 4: Calculate the 3D Delaunay triangle mesh of the distributed points.

Step 5: Construct a 3D Voronoi mesh surface with distributed points.

Step 6: Take the center of each 3D Voronoi grid surface as the scaling center, and appropriately scale each 3D Voronoi grid surface to form a grid frame.

Step 7: Use the scaled 3D Voronoi mesh surface to perform the subtraction calculation with the object surface.

Step 8: Obtain the finished hollow out surface.

Step 9: Thicken these hollowed out surfaces to obtain finished hollowed out entities.

Step 10: Integrate the main body of the object and the hollow out entity to complete the creative product object.

Figure 13 shows the execution flow of the entire project. 


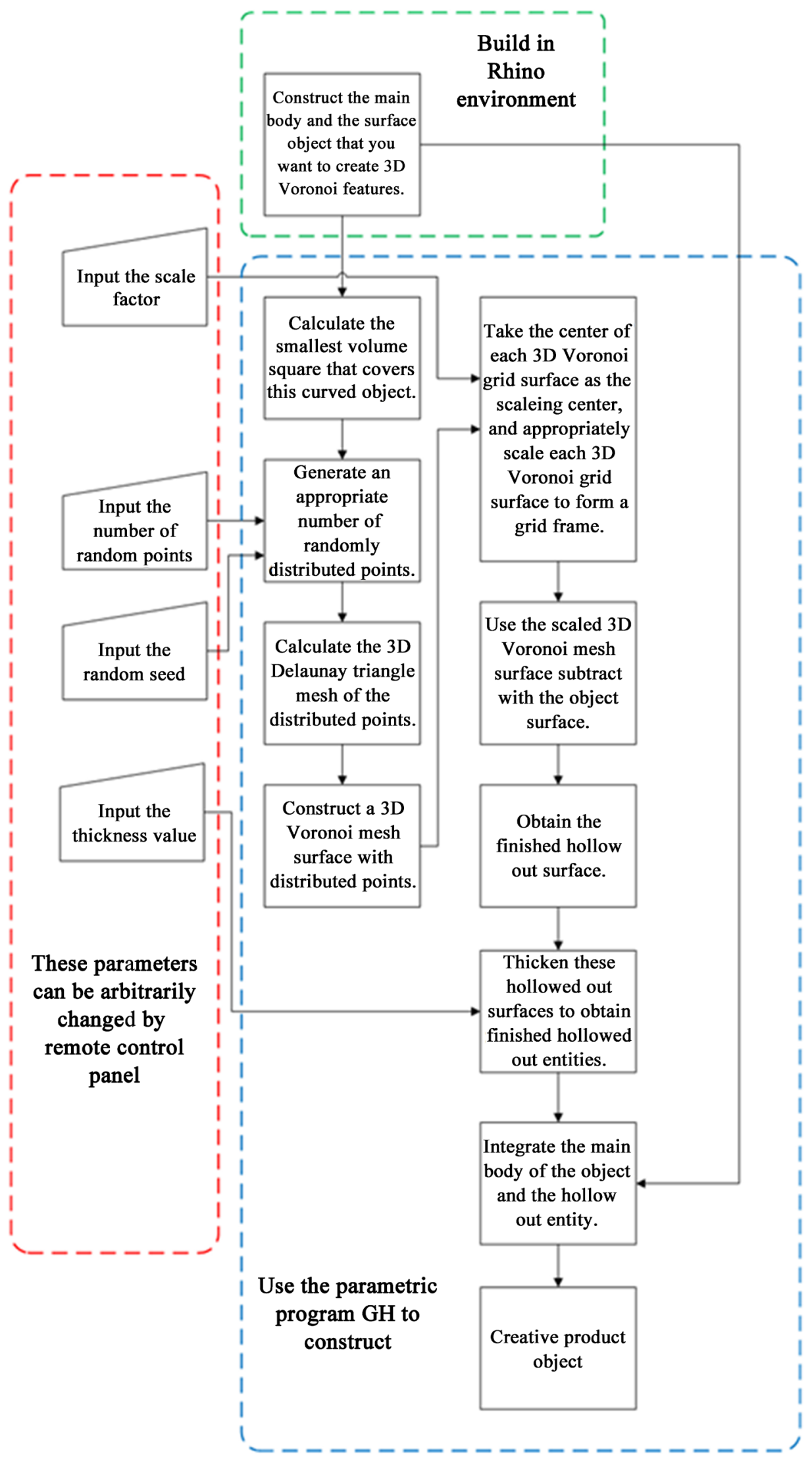

Figure 13. Flow chart of modeling in this article. 


\section{Conclusions}

This thesis is mainly in view of the importance of hollow out art in Chinese cultural heritage, and the 3D hollow out production craft has gradually lost. Therefore, the Delaunay triangle is constructed based on the Convex Hull interpolation algorithm, and the Voronoi Diagram feature is constructed based on the Divide and Conquer algorithm. And with Rhino modeling software as the main body, combined with the application of the parametric plug-in design program $(\mathrm{GH})$, the $3 \mathrm{D}$ models of the parametric creative hollow pen holder and the parametric creative hollow lampshade were respectively completed. The traditional craftsmanship is integrated into the modern manufacturing process with innovative techniques, and the Chinese cultural spirit and beauty of nature are successfully connected. Figure 14 shows a parametric creative hollow out pen holder constructed with random points of 100, 200 and 300. And Figure 15 shows the parametric creative hollow out lampshade constructed with random points of 100, 150 and 200.

Figure 16 is the $3 \mathrm{~d}$ printed product of the parametric creative hollow out pen holder, and its usage scenario. And Figure 17 is the $3 \mathrm{~d}$ printed product of the parametric creative hollow out lampshade and its usage scenario.

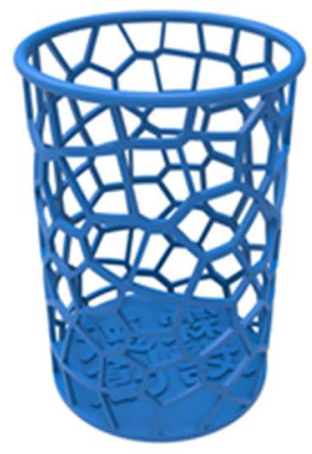

Random points 100

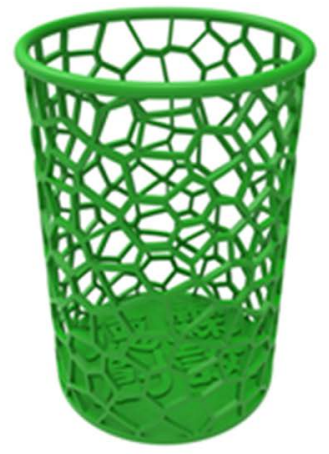

Random points 200

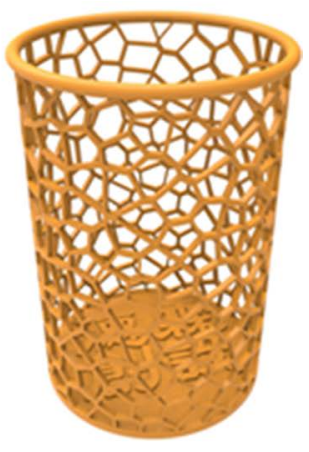

Random points 300

Figure 14. The parameterized creative hollow pen holder constructed with random points of 100, 200 and 300.

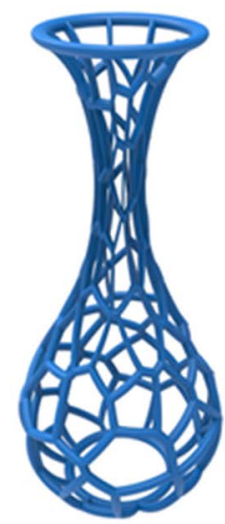

Random points 100

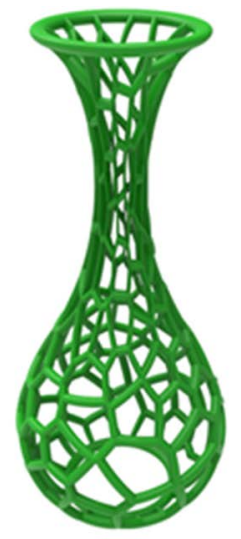

Random points 150

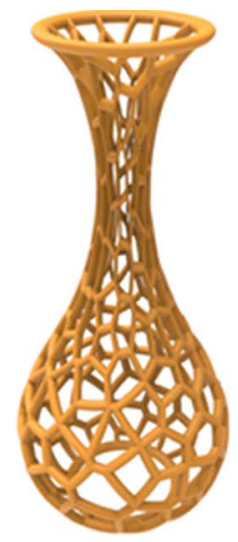

Random points 200

Figure 15. The parameterized creative hollow lampshade constructed with random points of 100,150 and 200. 

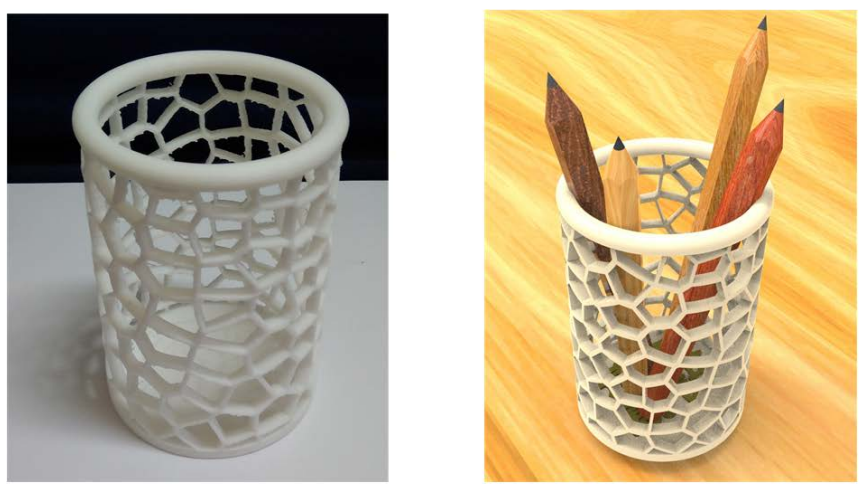

Figure 16. The $3 \mathrm{~d}$ printed product of the parametric creative hollow out pen holder and its usage scenario.
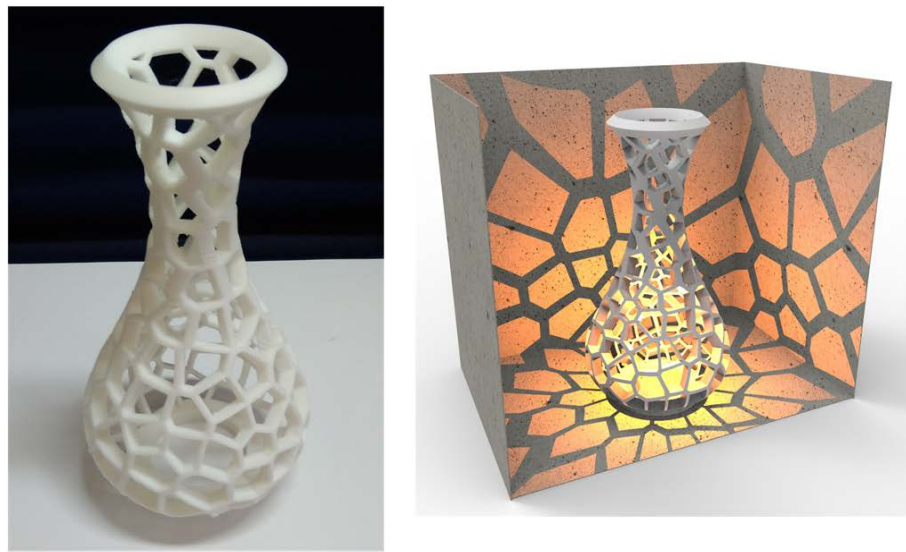

Figure 17. The $3 \mathrm{~d}$ printed product of the parametric creative hollow out lampshade and its usage scenario.

\section{Acknowledgements}

This research is grateful to the Ministry of Science and Technology for the funding support of the special research project for college students (106-2813-C237-001-H), which enabled the successful completion of this research.

\section{Conflicts of Interest}

The author declares no conflicts of interest regarding the publication of this paper.

\section{References}

[1] Peng, Y.-J. (2018) The Application of Generative Pattern in Product Surface. Master thesis, National Chiao Tung University.

[2] Zhuang, B.-H. (1980). Baptism of Folk Art. Lion Book Company, Taipei.

[3] Jiang, K.-W. (1988) The Essence of Paper-cutting Art. Hengyang Printing Co., Ltd., Taipei.

[4] Huang, B.-F. (1980) Western Silhouette Pattern Collection. Art Book Company, Taipei. 
[5] Chen, B.-Y. (1987). Chinese Folk Paper-Cutting. Wuling Publishing House, Taipei.

[6] You, S.-B. (2012) Nature's Patterns: Using Parametric Design as a Tool in Product Modeling. Master Thesis, National Chiao Tung University.

[7] Edelsbrunner, H. (1987) Algorithms in Combinational Geometry. Springer-Verlag, Heidelberg. https://doi.org/10.1007/978-3-642-61568-9

[8] Aurenhammer, F. (1991) Voronoi Diagrams: A Study of a Fundamental Geometric Data Structure. ACM Computing Surveys, 23, 345-405. https://doi.org/10.1145/116873.116880

[9] Kolahdouzan, M. and Shahabi, C. (2004) Voronoi-Based K nearest Neighbor Search for Spatial Network Databases. Proceedings of the Thirtieth International Conference on Very Large Data Bases, Toronto, 31 August 2004, 840-851. https://doi.org/10.1016/B978-012088469-8.50074-7

[10] Ajani, S. (2013) An Efficient Approach for Clustering Uncertain Data Mining Based on Hash Indexing and Noronoi Clustering. Proceedings of the International Conference on Computational Intelligence and Communication Networks, Washington DC, 27-29 September 2013, 5-9. https://doi.org/10.1109/CICN.2013.106

[11] Paper, C. and Queen, K.I. (2008) Distributed Voronoi Diagram Computation in Wireless Sensor Networks Distributed Voronoi Diagram Computation in Wireless Sensor Networks. Proceedings of the Twentieth Annual Symposium on Parallelism in Algorithms and Architecture, Munich, 14-16 June 2008, 364-374.

[12] Li, S.-Y. (2013) Using Parametric Design in Product Creation. Master Thesis, National Chiao Tung University. 